\title{
Ileal neuroendocrine tumor metastasis to pancreas and liver: A case report
}

\author{
Michael Sean Bleszynski, David F. Schaeffer, Maja Segedi
}

\begin{abstract}
Introduction: Neuroendocrine tumors (NETs) formally known as carcinoids tumors are neoplasms that arise from enterochromaffin cells. The NETs most commonly occur in the gastro-intestinal tract and predominately within the ileum. Symptomatic ileal NETs have most likely metastasized to the liver at the time of diagnosis. Pancreatic metastasis from ileal NETs are exceptionally rare. Case Report: A 76-year-old female with a primary ileal carcinoid underwent right hemi-colectomy secondary to bowel obstruction. One-year post bowel resection, the patient presented with carcinoid syndrome, imaging revealed a singular hepatic metastasis (segment 2) and a pancreatic tail mass. After radiologic workup with an abdominal CT, PET and octreotide scan a presumed diagnosis of recurrent metastatic NET was made. Multi-disciplinary rounds discussed the case. Laparoscopic distal pancreatectomy, splenectomy and radiofrequency ablation of the liver tumor were performed. Surgical pathology of the pancreatic mass was consistent with primary ileal carcinoid tumor. Conclusion: Ileal NET metastasis to the pancreas is a rare
\end{abstract}

Michael Sean Bleszynski ${ }^{1}$, David F. Schaeffer ${ }^{2}$, Maja Segedi ${ }^{1}$ Affiliations: ${ }^{1}$ Department of General Surgery, University of British Columbia, Vancouver, Canada; ${ }^{2}$ Department of Pathology, University of British Columbia, Vancouver, Canada. Corresponding Author: Michael Bleszynski, MD, The University of British Columbia Faculty of Medicine, Department of Surgery, Division of General Surgery, Vancouver General Hospital Rm 3100 - Jim Pattison Pavillion North, 950 West 10th Avenue, Vancouver, BC, V5Z 1M9 Canada; E-mail: mbleszyn@gmail.com

Received: 27 November 2016

Accepted: 07 February 2017

Published: 07 March 2017 phenomenon. Metastatic NET management should be discussed with a multi-disciplinary team. Ro resection of primary and metastatic NETs offers the only chance of cure. Locoregional, systemic therapies, and surgical debulking can offer a survival benefit in unresectable disease or in cases where resection incurs a high perioperative risk.

Keywords: Ileum, Metastasis, Neuroendocrine tumor, Pancreas

\section{How to cite this article}

Bleszynski MS, Schaeffer DF, Segedi M. Ileal neuroendocrine tumor metastasis to pancreas and liver: A case report. Int $J$ Hepatobiliary Pancreat Dis 2017;7:11-14.

Article ID: 100066IJHPDMB2017

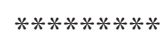

doi:10.5348/ijhpd-2017-66-CR-2

\section{INTRODUCTION}

The overall incidence of neuroendocrine tumors (NETs) in the US is approximately $1-2$ cases per 100,000 people [1]. Neuroendocrine tumors were previously called carcinoids. However, the nomenclature of carcinoid tumor still exists within literature. The NETs are neoplasms that develop from enterochromaffin cells, which can secrete active substances such as serotonin and bradykinin that may bypass portal circulation resulting in carcinoid syndrome [2]. Carcinoid syndrome occurs in approximately $10 \%$ of patients with jejunal-ileal NETs and is characterized by a combination of symptoms 
ranging from; flushing, chronic diarrhea, abdominal pain, carcinoid heart disease and bronchial constriction [3].

Approximately $70 \%$ of NETs are located in the gastrointestinal tract with tumor location most commonly found in the small bowel (45\%), rectum (20\%), appendix (17\%), colon (11\%) and stomach (7\%) [4]. Gastrointestinal NETs most commonly occur at the ileum and $6-30 \%$ of ileal NETs will have symptoms of carcinoid syndrome [5]. Ileal NETs presenting with carcinoid syndrome are associated with hepatic metastasis in more than $95 \%$ of cases [5]. Hepatic metastases are most common while peritoneum, bone and ovaries are other rare documented sites of distant metastasis $[6,7]$.

We currently have not identified any case reports that have discussed a primary ileal carcinoid tumor metastasizing to the pancreas with concurrent hepatic metastasis.

\section{CASE REPORT}

Patient's informed consent was provided. A 76-yearold female presented in 2015 with worsening flushing and diarrhea one-year post right hemi-colectomy for a primary low-grade ileal neuroendocrine tumor. Initially, she developed recurrent small bowel obstructions, which were treated nonoperatively. Investigation with colonoscopy identified a lesion at the terminal ileum, which led to the decision for right hemicolectomy. Surgical pathology post hemicolectomy showed a T2N1 low grade neuroendocrine tumor, 4/12 lymph nodes positive, Ki-67 < 5\% and negative margins. No adjuvant chemotherapy was prescribed. Serum chromogranin A level post hemicolectomy was 94 units and one year later had risen to 176 units, concurrent with progressive flushing and diarrhea.

Follow-up investigations included PET, CT and octreotide scans. PET scan identified a new liver lesion in segment $2 / 3$ and a lesion in the body/tail of the pancreas, with the pancreatic lesion consistent with neuroendocrine tumor. Tri-phasic abdominal CT scan showed a mildly hyper-enhancing lesion at the pancreatic tail measuring $1.9 \mathrm{~cm}$. No definite evidence of a locally recurrent mass at the previous surgical site was seen on imaging. Despite new lesions identified on CT scan and PET scan, a subsequent octreotide scan did not identify any convincing octreotide avid abnormality.

Past medical history was significant for arthritis. Previous surgical history not yet mentioned includes laminectomy/disc surgery (2013), spinal stenosis surgery (2012) and remote laparoscopic cholecystectomy. Regular home medications consisted of Voltaren and a daily proton pump inhibitor. Known allergies were to sulfa drugs. Patient was married and had an active lifestyle playing golf regularly.

The case was discussed at our institutional multidisciplinary liver tumor rounds. It was concluded that the new pancreatic and liver lesions would be best managed surgically. Consent was obtained for laparoscopic distal pancreatectomy with possible splenectomy and radiofrequency ablation of the liver lesion. It was felt that resecting the liver lesion at the same time as performing the pancreatic resection would incur too high of a perioperative complication risk for this patient at this time.

A laparoscopic distal pancreatectomy, splenectomy, and intraoperative radiofrequency ablation of the liver lesion (segment 2) were performed without complications. Patient recovered well from surgery and was discharged home on postoperative day-6.

Surgical Pathology report of the resected pancreatic mass and parenchyma revealed a metastatic (midgut) ileal neuroendocrine tumor with the following characteristics;

- Single focus within pancreatic tail, size $=2.5 \mathrm{~cm}$

- Histological grade 2

- Mitotic rate: up to 3 mitosis/10 hpf

- Ki67 labeling index: up to 4\% (Figure 1A)

- Positive for lymphovascular invasion

- Surgical resection margins negative for malignancy

- 2/10 peripancreatic lymph nodes positive for metastatic neuroendocrine tumor

Hematoxylin and eosin stain of resected pancreatic mass pictured in Figure 1B. Staining: Positive for synaptophysin, chromogranin A (Figure $1 \mathrm{C}$ ), CDX2, serotonin and negative for PAX8.

\section{DISCUSSION}

We are able to deduce that this was a recurrent ileal NET, which had metastasized to the pancreas, based pathologic examination of the pancreatic specimen. Positive CDX2 is commonly associated with colorectal adenocarcinoma, however, positive CDX2 has also been associated with neoplasms with intestinal differentiation such as gastrointestinal NETs [8]. PAX8 is expressed in pancreatic islet cells, thyroid tissue and reproductive systems. PAX8 is able to distinguish between pancreatic NETs and ileal NETs [9]. If the pancreatic tumor was a primary NET, PAX8 staining would have been positive.

Metastatic lesions (of any tumor type) to the pancreas are quite rare, composing approximately $2 \%$ of all pancreatic malignancies; a systemic review has shown that pancreatic metastasectomy provides a survival benefit in select cases [10]. One retrospective review of tumors with metastasis to the pancreas identified one case of an ileal NET amongst other primary tumors [11].

Surgery is the only definitive treatment modality for curative intent of NETs, but is also used for palliative control of symptoms via cytoreductive procedures [6]. Although not curative, surgical ablation therapy is a utilized technique directed at metastatic hepatic NETs for symptom control [12]. There are several radiological options available (CT, MR, PET, octreotide scan) for 

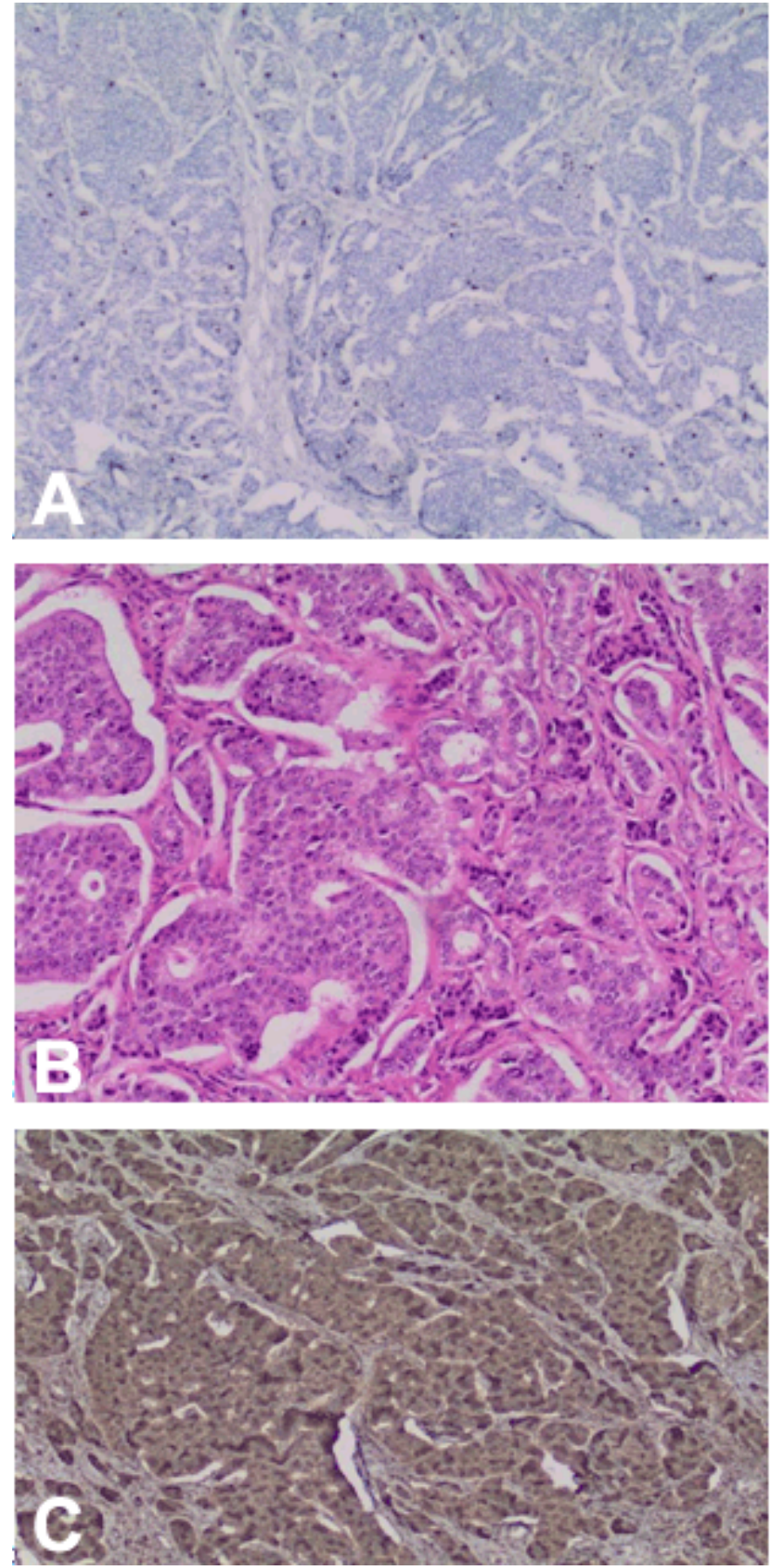

Figure 1A-C: Resected pancreatic mass. Magnification A: Ki-67 stain, x400, B: H\&E stain, x100, C: Chromogranin stain, x400.

the diagnosis and evaluation of NETs for potential surgical resection. Each of these techniques has different strengths and there is a cost association that must be taken into consideration. Furthermore, with different surgical options at a surgeon's disposal, multidisciplinary rounds are essential for providing optimal care.

\section{CONCLUSION}

Small bowel neuroendocrine tumor (NET) most commonly metastasize to the liver, and metastases to the pancreas is a rare phenomenon. We describe a case of a previously resected well-differentiated ileal NET that metastasized one year later to the pancreas. After radiologic work up, the case was brought forth to a multidisciplinary team and consensus decision was to undergo surgical management. Via laparoscopy, distal pancreatectomy, splenectomy and radiofrequency ablation of the involved liver lesion were successfully performed. Management of metastatic NETs should be discussed with a multidisciplinary team.

$* * * * * * * * *$

\section{Author Contributions}

Michael Sean Bleszynski - Substantial contributions to conception and design, Acquisition of data, Analysis and interpretation of data, Drafting the article, Revising it critically for important intellectual content, Final approval of the version to be published

David F. Schaeffer - Analysis and interpretation of data, Revising it critically for important intellectual content, Final approval of the version to be published

Maja Segedi - Analysis and interpretation of data, Revising it critically for important intellectual content, Final approval of the version to be published

\section{Guarantor}

The corresponding author is the guarantor of submission.

\section{Conflict of Interest}

Authors declare no conflict of interest.

\section{Copyright}

(C) 2017 Michael Sean Bleszynski et al. This article is distributed under the terms of Creative Commons Attribution License which permits unrestricted use, distribution and reproduction in any medium provided the original author(s) and original publisher are properly credited. Please see the copyright policy on the journal website for more information.

\section{REFERENCES}

1. Kulke $\mathrm{MH}$, Mayer RJ. Carcinoid tumors. N Engl J Med 1999 Mar 18;340(11):858-68.

2. Salyers WJ, Vega KJ, Munoz JC, Trotman BW, Tanev SS. Neuroendocrine tumors of the gastrointestinal tract: Case reports and literature review. World J Gastrointest Oncol 2014 Aug 15;6(8):301-10.

3. Poncet G, Faucheron JL, Walter T. Recent trends in the treatment of well-differentiated endocrine carcinoma of the small bowel. World J Gastroenterol 2010 Apr 14;16(14):1696-706.

4. Maggard MA, O'Connell JB, Ko CY. Updated population-based review of carcinoid tumors. Ann Surg 2004 Jul;240(1):117-22.

5. Scherübl H, Jensen RT, Cadiot G, Stölzel U, Klöppel G. Neuroendocrine tumors of the small bowels are 
on the rise: Early aspects and management. World J Gastrointest Endosc 2010 Oct 16;2(10):325-34.

6. Maroun J, Kocha W, Kvols L, et al. Guidelines for the diagnosis and management of carcinoid tumours. Part 1: The gastrointestinal tract. A statement from a canadian national carcinoid expert group. Curr Oncol $2006 \operatorname{Apr} ; 13(2): 67-76$

7. Landerholm K, Falkmer S, Järhult J. Epidemiology of small bowel carcinoids in a defined population. World J Surg 2010 Jul;34(7):1500-5.

8. Saad RS, Ghorab Z, Khalifa MA, Xu M. CDX2 as a marker for intestinal differentiation: Its utility and limitations. World J Gastrointest Surg 2011 Nov 27;3(11):159-66.

9. Sangoi AR, Ohgami RS, Pai RK, Beck AH, McKenney JK, Pai RK. PAX8 expression reliably distinguishes pancreatic well-differentiated neuroendocrine tumors from ileal and pulmonary well-differentiated neuroendocrine tumors and pancreatic acinar cell carcinoma. Mod Pathol 2011 Mar;24(3):412-24.

10. Adler H, Redmond CE, Heneghan HM, et al. Pancreatectomy for metastatic disease: A systematic review. Eur J Surg Oncol 2014 Apr;40(4):379-86.

11. Moussa A, Mitry E, Hammel P, et al. Pancreatic metastases: A multicentric study of 22 patients. Gastroenterol Clin Biol 2004 Oct;28(10 Pt 1):872-6.

12. Mayo SC, Herman JM, Cosgrove D, et al. Emerging approaches in the management of patients with neuroendocrine liver metastasis: Role of liverdirected and systemic therapies. J Am Coll Surg 2013 Jan;216(1):123-34.
Access full text article on other devices

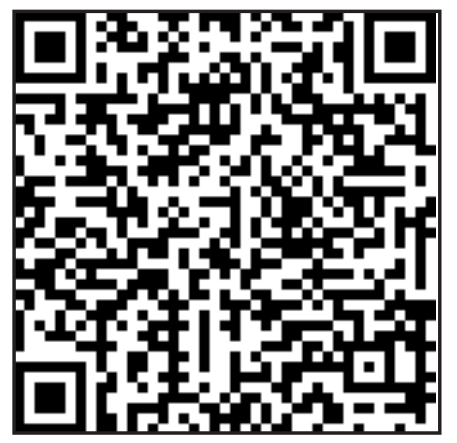

Access PDF of article on other devices

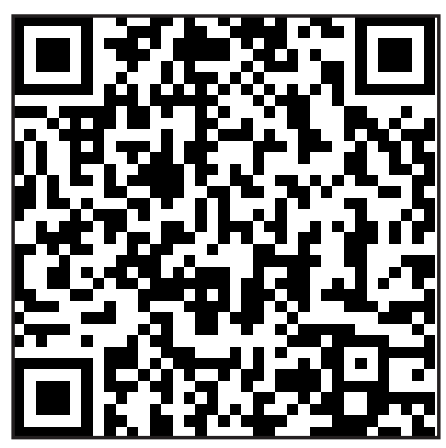

